PRAXIS

ecucativa

Universidad Nacional de La Pampa

Facultad de Ciencias Humanas

Instituto de Ciencias de la Educación

para la investigación interdisciplinaria



ISSN 2313-934X

SANTA ROSA, LA PAMPA, ARGENTINA

Correo electrónico: iceii@humanas.unlpam.edu.ar

Disponible en https://cerac.unlpam.edu.ar/index.php/praxis

Trabajo docente y capitalismo académico. Girando las lentes para comprender el trabajo cotidiano en la universidad contemporánea. Artículo de Verónica Soledad Walker. Praxis educativa, Vol. 25, No 3 septiembre - diciembre 2021. E - ISSN 2313-934X. pp. 1-18. https://dx.doi.org/10.19137/praxiseducativa-2021-250306

Esta obra se publica baja Licencia Creative Commons 4.0 Internacional CC BY-NC-SA Atribución, No Comercial, Compartir igual

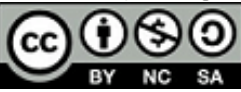

\title{
Trabajo docente y capitalismo académico. Girando las lentes para comprender el trabajo cotidiano en la universidad contemporánea
}

Teaching work and academic capitalism. Turning the lenses to understand the daily work in the contemporary university

Trabalho docente e capitalismo acadêmico. Virando as lentes para entender o cotidiano de trabalho na universidade contemporânea

\section{Verónica Soledad Walker}

Universidad Nacional del Sur

Argentina

veroswalker@gmail.com

ORCID: 0000-0002-3861-7108 


\section{Resumen}

La introducción de los principios de la Nueva Gestión Pública en la educación superior a nivel mundial provocó profundas transformaciones en los sistemas, las instituciones y las prácticas de los actores. El trabajo docente universitario sufrió modificaciones significativas en sus condiciones, formas de organización y evaluación. En este escrito, se focaliza en las formas que asume el trabajo docente en el contexto del capitalismo académico a partir de la comprensión de aquello que circula en la cotidianeidad de la vida universitaria. Para ello, se recuperan fragmentos de entrevistas y extractos de conversaciones informales con docentes de universidades públicas de Argentina. Se abordan cuestiones como el reconocimiento material y simbólico del trabajo realizado, las relaciones entre trabajo docente y vida cotidiana, la cuestión del tiempo de trabajo como ordenador del tiempo de la vida, la intensificación de las tareas y la creciente demanda de disponibilidad permanente.

Palabras clave: universidad; nueva gestión pública; trabajo docente, vida cotidiana, sentidos

\section{Abstract}

The introduction of the principles of New Public Management in higher education worldwide caused profound transformations in the systems, institutions and practices of the actors. University teaching work underwent significant modifications in its conditions, forms of organization and evaluation. In this writing we focus on the forms that teaching work assumes in the context of academic capitalism from the understanding of what circulates in the daily life of the university. For this, fragments of interviews and extracts of informal conversations with professors of public universities in Argentina are recovered. Issues such as the material and symbolic recognition of the work done, the relationships between teaching work and daily life, the question of working time as the computer of life time, the intensification of tasks and the growing demand for permanent availability are addressed.

Keywords: university; New Public Management; teaching work; daily life; meanings.

\section{Resumo}

A introdução dos princípios da Nova Gestão Pública no ensino superior mundial causou profundas transformações nos sistemas, instituições e práticas dos atores. 0 trabalho docente universitário sofreu modificações significativas nas suas condições, formas de organização e avaliação. Nesta escrita, enfoca as formas que o trabalho docente assume no contexto do capitalismo acadêmico a partir da compreensão do que circula no cotidiano universitário. Para isso, são recuperados fragmentos de entrevistas e trechos de conversas informais com professores de universidades públicas da Argentina. São abordadas questões como o reconhecimento material e simbólico do trabalho realizado, as relações entre o trabalho docente e o

cotidiano, a questão do tempo de trabalho como computador da vida, a intensificação das tarefas e a crescente demanda por disponibilidade permanente.

Palavras-chave: universidade, Nova Gestão Pública, trabalho docente, vida quotidiana, significados 


\section{Introducción}

En las últimas décadas, el campo de la educación superior a nivel mundial se encuentra atravesado por tendencias globales que operan como reguladores de las dinámicas institucionales y las prácticas de los sujetos. Se trata de cambios orientados por los principios de la Nueva Gestión Pública (Verger y Normand, 2015) en el marco de nuevas relaciones entre Estado, sociedad y educación superior (Sousa, 2005). En el caso del sector universitario, las denominaciones "universidad comercial", "universidad controlada" y "universidad auditada" (Barnett, 2002) dan cuenta de un desplazamiento del eje de coordinación de los sistemas de educación superior desde el gobierno y las corporaciones académicas -en general, la esfera pública- hacia el mercado.

El concepto de "capitalismo académico", acuñado por Slaughter y Leslie (1997), refiere a los comportamientos de mercado dentro de las universidades y sus claustros como la competencia institucional, la búsqueda de fondos y la venta de servicios (Saforcada y Rodríguez Golisano, 2019). El ingreso de la universidad a la fase del capitalismo académico supuso la hegemonía de una visión de sus funciones y propósitos reducidos a la prestación de servicios y del papel del Estado como órgano de control de resultados y racionalización de recursos (Ibarra Colado, 2003). El desarrollo de la dimensión comercial y la reducción de financiamiento estatal a las universidades conlleva la puesta en marcha de mecanismos que fuerzan a las instituciones a competir entre sí por los estudiantes, el personal académico y reputaciones, y a financiar sus actividades por medio de recursos de una diversidad de fuentes fiscales y privadas, obtenidos de manera competitiva. Tales mecanismos suponen una reestructuración de la institución universitaria a nivel del sistema, las instituciones y las prácticas de los actores.

Entre estos últimos, el personal docente ha visto significativamente modificadas las condiciones, formas de organización y evaluación de su trabajo en la universidad. Las actuales formas de contratación laboral caracterizadas por el tiempo parcial y los contratos temporarios, el desarrollo de carreras nómades, la virtualización de las actividades, la intensificación de tareas y el control de los resultados forman parte de las nuevas reglas de juego de la universidad contemporánea.

En este escrito, se busca poner el foco en las formas que asume el trabajo docente en el contexto del capitalismo académico a partir de la comprensión de aquello que circula en la cotidianeidad de la vida universitaria. Se abordan cuestiones como el reconocimiento material y simbólico del trabajo realizado, las relaciones entre el trabajo docente y la vida cotidiana, sus efectos en esferas como la salud, la familia, la recreación, etc.; la cuestión del tiempo de trabajo como ordenador del tiempo de la vida, la intensificación de las tareas y la dimensión colectiva del trabajo. El escrito se estructura en tres apartados. En primer lugar, se explicitan los referentes conceptuales y las decisiones metodológicas que orientaron el estudio. La identificación del giro epistemológico en los estudios sobre la universidad en general y el trabajo docente en particular junto a la recuperación de las categorías de vida cotidiana, experiencia universitaria y sentidos, justifican la estrategia de tomar las voces de los actores como punto de partida para el análisis. En segundo lugar, sobre la base de las consideraciones teórico-metodológicas efectuadas, se parte de 
fragmentos de entrevistas y extractos de conversaciones informales y comunicaciones institucionales que permiten identificar aspectos relevantes de la cotidianeidad del trabajo docente en la universidad contemporánea: los vínculos entre tendencias globales y esfuerzos personales, la creciente demanda de vivir online, las relaciones entre vida laboral y vida personal y la centralidad del tiempo en la vida y el trabajo. En tercer lugar, y para finalizar, se esboza una serie de conclusiones preliminares que invitan a la apertura de nuevas líneas de indagación sobre el tema.

\section{Consideraciones teórico-metodológicas}

La revisión de la literatura especializada permite afirmar que, en la década de 1980, comienzan a difundirse a nivel internacional producciones sobre la universidad entendida como "organización compleja", "anarquía organizada" o "sistema pesado en la base" (Krotsch, 2001) y sobre el profesorado universitario y sus espacios de trabajo. Estudios pioneros como los de Clark (1983), Becher (1989), Perkin (1984) y Boyer et al. (1994) colocan, desde la categoría de profesión, a los académicos como objeto de investigación y actores clave en el análisis de los sistemas de educación superior. En aquellas primeras contribuciones, el académico fue definido como miembro de una comunidad o profesión cuya razón de ser es su capacidad de generar y transmitir conocimiento en una institución atravesada por la dimensión disciplinaria (Clark, 1983).

En América Latina, en la misma década, los estudios de Brunner (1985) y Brunner y Flisfisch (1989) señalan la constitución de un mercado ocupacional académico a partir del proceso de masificación de las universidades iniciado en la década de 1960 y las consecuentes modificaciones en las condiciones de trabajo del mundo académico. Estos cambios cuantitativos (por el incremento numérico de los grupos de docentes que debieron satisfacer la demanda educativa de amplios sectores estudiantiles) y cualitativos (por la nueva división social del trabajo intelectual) produjeron un nuevo tipo de profesional, "un hombre que no necesariamente vive para la cultura o el conocimiento pero que de cualquier modo vive de la cultura" (Brunner, 1985, p. 6).

En Argentina, la expansión no planificada del cuerpo docente, a partir de la apertura democrática de 1983, transformó radicalmente sus características en términos de perfil socioeconómico y prácticas individuales e institucionales (Marquina, 2013). Este fenómeno confluyó, por otra parte, con las dificultades experimentadas por muchos profesionales para insertarse en el mercado ocupacional, que hizo de la universidad un refugio ocupacional para sus propios graduados, especialmente los provenientes de aquellas disciplinas cuyo campo ocupacional extrauniversitario resulta más recortado y complejo (Chiroleu, 2002).

Las políticas universitarias implementadas a partir de la década de 1990, especialmente las de evaluación, apoyadas en nociones de eficiencia, productividad y rendimiento, provocaron cambios profundos en la cultura académica y las condiciones de producción intelectual (Naidorf, 2009), las dinámicas institucionales y las formas de organización del trabajo en las universidades públicas (Araujo, 2003; García de Fanelli, 2008; Llomovatte y Wischnevsky, 1998; Tello, 2008).

Estos cambios se enmarcan en un conjunto de tendencias que atraviesan a los sistemas de educación superior a nivel mundial, derivadas de las demandas de los contextos económico, político 
y social a las universidades y de los cambios en el mundo del trabajo en general, que adoptan formas específicas según los ámbitos nacionales, institucionales y disciplinares en los que se despliegan (Walker, 2020). Entre estas tendencias, se pueden mencionar las nuevas modalidades de contratación laboral, formas de organización y evaluación del trabajo académico (Ibarra Colado, 2003; Gill, 2015; Musselin, 2007; Robertson, 2013), el aumento de las actividades de internacionalización, los cambios en las carreras académicas y la configuración de carreras nómades (Enders y Kaulisch, 2006), así como la creciente virtualización de las actividades, la expansión de los posgrados y el avance del credencialismo (Gil Antón, 2013; Gill, 2015; Silva Júnior y Sguissardi, 2013). De esta manera, los estudios referenciados permiten pensar los cambios en el trabajo docente universitario en relación con los cambios operados en el mundo del trabajo en general. Desde esta perspectiva, entonces, las universidades son consideradas como espacios laborales y los/as docentes como trabajadores/as (Allmer, 2018).

La revisión de la literatura sobre el tema permite advertir que, durante la última década del siglo XX, en el campo de producción de conocimientos sobre la educación superior, se asistió a una mutación de estudios históricos y normativos sobre los sistemas hacia investigaciones centradas en la dimensión organizacional de las instituciones. Este viraje habilitó el despliegue posterior de perspectivas interpretativas de la vida cotidiana y las experiencias de los sujetos en el mundo universitario. A partir de la primera década del siglo XXI, tuvo lugar un giro epistemológico basado en la comprensión de la dimensión subjetiva y experiencial a partir de lo que viven, sienten y piensan quienes transitan la universidad. Actualmente, se reconoce que, si bien es necesaria una perspectiva estructural para identificar las tendencias de la educación superior y realizar miradas comparadas, resulta imprescindible la comprensión de las singulares experiencias universitarias que se configuran en la relación entre biografía e institución en un tiempo histórico determinado (Carli, 2016). Como sostiene Wright Mills (1994), en ese punto donde converge la biografía y la estructura es que tiene lugar la imaginación sociológica.

El concepto de vida cotidiana cobra especial centralidad para este tipo de análisis. Lo cotidiano es definido como todo tipo de actividades que constituyen, desde cada sujeto particular, procesos significativos de reproducción social y apropiación cultural. En su Sociología de la vida cotidiana, Heller (1987) conceptualiza lo cotidiano como una dimensión de cualquier situación social cuyo eje es el trabajo humano como articulador de la reproducción individual (en la familia, la comunidad, la vida privada) y la reproducción social. Desde esta perspectiva, la vida cotidiana en la universidad es concebida como un tiempo-espacio presente, no rutinario (no es lo que sucede todos los días, sino lo que puede suceder cualquier día dentro del horizonte de posibilidades) propio de las tramas construidas entre personas quienes, al encontrarse unas con otras, crean un cierto espacio común (Rockwell, 2018).

En este escrito, interesa focalizar en la experiencia de trabajo docente en la universidad contemporánea. Se parte de considerar que la posición que cada docente ocupa en el espacio universitario y desde la cual desarrolla su trabajo cotidiano constituye la trama material desde la que se construyen los sentidos sobre su trabajo. La manera en que cada docente siente, piensa y 
significa su trabajo es resultado de su experiencia vivida, del sentido subjetivo construido en su quehacer cotidiano en el marco de relaciones objetivas que condicionan sus prácticas (Bourdieu, 2008). Desde esta perspectiva estructural constructivista, que recupera la sociología comprensiva weberiana, se entiende por sentido el significado, los motivos y el valor que los sujetos atribuyen a su acción. Si bien el sentido es social e intersubjetivamente construido, no es único ni idéntico para todos, incluso para quienes pertenecen a un mismo grupo u ocupan posiciones similares. Las experiencias vividas por actores diferencialmente posicionados en el espacio social crean y recrean sentidos disímiles y hasta contradictorios sobre las "cosas sociales". Y esos sentidos se expresan en lo que se dice, lo que se hace y lo que se dice que se hace; se ponen de manifiesto en la praxis cotidiana y en el discurso que se utiliza para hablar de ella (Beltrán Villalva, 2013).

Para llevar adelante el análisis, se parte del material empírico recogido en investigaciones finalizadas y en curso desarrolladas durante los últimos diez años. Por un lado, se recuperan extractos de entrevistas semiestructuradas realizadas a docentes de universidades públicas argentinas realizadas en el marco de proyectos de investigación ya culminados. ${ }^{.}$Se trata de un corpus de cuarenta y dos entrevistas a docentes de distintas pertenencias institucionales y disciplinares, diferentes categorías, dedicación horaria, forma de contratación laboral y antigüedad en la docencia universitaria.

En coherencia con el paradigma interpretativo asumido y el abordaje metodológico cualitativo, no se procura un criterio de representatividad de los discursos analizados, sino de significatividad. Recuperando a Guber (2004), el concepto de representatividad, vinculada a la dimensión numérica, establece una asociación entre representatividad y generalización explicativa con magnitud cuantitativa de la muestra. Pero otra posibilidad es que una muestra sea significativa, esto es, que un hecho o un caso sean pertinentes para dar cuenta de cierto haz de relaciones en un sistema social. De esta manera, un acontecimiento no es más o menos válido para la investigación únicamente si se presenta tantas veces - procedimiento que, sin embargo, no habría que descuidar-, sino que los hechos, las prácticas, las verbalizaciones, etc., interesan especialmente por su integración en un sistema de significados y de relaciones sociales. Por eso, siguiendo a la autora, fue fundamental el criterio de significatividad para la construcción de la muestra de docentes atendiendo a la diversidad de posiciones en el espacio universitario (diferente categoría docente, dedicación, pertenencia disciplinar, género, antigüedad, forma de contratación, etc.). Las entrevistas semiestructuradas (Sautu, 2005) fueron el instrumento privilegiado para conocer los sentidos atribuidos al trabajo cotidiano en la universidad.

Por otro lado, se recogen y analizan expresiones y relatos de colegas formulados en diversas situaciones informales de la vida universitaria en los últimos cinco años. Se trata de decires y sentires compartidos en conversaciones cotidianas entre docentes, contenido de las comunicaciones institucionales recibidas a diario, mensajes de correo electrónico, etc. Es decir, expresiones de esa trama discursiva que, en su dimensión simbólica, visibiliza la dimensión material en la que se despliega el trabajo cotidiano. A partir de estas narrativas, se propone realizar un 
ejercicio reflexivo consistente en el pasaje de una forma de investigación "sobre" el trabajo docente hacia un modo de investigar "desde" y "con" los y las docentes.

\section{Girando las lentes: escenas de la vida cotidiana en la universidad}

¿Qué significaría girar nuestras lentes sobre los procesos de trabajo, la gobernanza institucional y las condiciones de producción? ¿Qué podríamos encontrar si, en vez de estudiar a otros, centráramos nuestra mirada en nuestra propia comunidad, y tomáramos como datos no la pulcra publicación o la charla de bella factura, sino el flujo interminable de comunicaciones y prácticas en las que todas y todos estamos inmersos y enredados, a menudo de mala gana? (Gill, 2015, p. 46)

Como se anticipó, en el presente apartado, se propone partir de fragmentos de ese flujo interminable de comunicaciones en que la comunidad académica está inmersa a diario. Se recuperan extractos de entrevistas, correos electrónicos y conversaciones informales entre colegas que resultan significativos para identificar y analizar distintas dimensiones que atraviesan la cotidianeidad de la experiencia de trabajo docente en la universidad contemporánea. ii

\section{Tendencias globales, esfuerzos personales}

Te mando este audio porque me llamó $X$ por lo que hablábamos el otro día, son poquitos estudiantes anotados y no podrían solventar económicamente el curso. Yo ya te manifesté el otro día mi opinión, yo lo daría igual por un tema de que igual nos sirve a nosotras para el CV. (Conversación por WhatsApp, Profesora, CSyH, 2019) Ahora, con la nueva resolución, la gestión está trabajando en el diseño del SIED (Sistema Institucional de Educación a Distancia). Me llamaron porque quieren que coordine el Área de Educación a Distancia (EaD) de la universidad, pero no hay un "mango". iii Sería ad honorem. Y dije que sí... es lo que siempre quise, me preparé para eso, hay que ocupar ese espacio, si no lo ocupa alguien que no tiene idea. Igual van a intentar que, con el tiempo, se pueda remunerar. (Conversación con Profesora, CSyH, 2019)

Ante la escasa respuesta que recibieron las convocatorias realizadas en febrero, marzo y mayo del corriente año, queremos solicitar la colaboración de las áreas para conformar el Consejo Asesor $X$ cuya mayor responsabilidad es la puntuación de la producción científica de los proyectos de investigación para asignar los subsidios anuales a cada grupo (...). En la convocatoria, hubo solo dos postulaciones. Teniendo en cuenta que hay 40 proyectos, las dos personas designadas deberán puntuar en el menor tiempo posible toda la producción anual de nuestros docentes e investigadores a efectos de que se puedan distribuir los subsidios que ya están disponibles. Dada la dificultad de dicha tarea, es previsible que tarden varios meses en poder terminarla y el dinero perderá valor. (Extracto de un mail institucional, $\mathrm{CSyH}, 2019$ ) 
Los tres fragmentos anteriores invitan a detener la mirada en tres aspectos de la vida universitaria que han cobrado relevancia en los últimos años: los posgrados, la educación a distancia (EaD) y la evaluación. En Argentina, la expansión de los posgrados, el desarrollo de la EaD y la hegemonía de políticas universitarias de evaluación de la calidad se sucedieron sin la asignación de presupuesto específico convirtiéndose en nuevas cargas para el personal docente.

Con respecto a los posgrados, su crecimiento exponencial a partir de la década de 1990 en Argentina no fue acompañado con la asignación de financiamiento con las dificultades que ello conlleva para el funcionamiento de los programas. El principio rector para el nivel es el autofinanciamiento, con prevalencia del financiamiento hacia la demanda que recae fundamentalmente en el estudiantado a través del cobro de matrículas y aranceles. También existen ofertas no aranceladas en universidades públicas que buscan garantizar el principio de gratuidad distintivo de los estudios superiores en Argentina, recurriendo a un financiamiento cruzado con el nivel de grado en cuanto a personal docente y administrativo, infraestructura y equipamiento (Walker, 2020). La carencia de una partida presupuestaria y las distintas modalidades de financiamiento a las que recurren los programas de posgrado promueve diversas formas de contratación de docentes. En algunos casos, la enseñanza de posgrado es parte del cumplimiento del cargo docente en el grado, aunque en la mayoría de la oferta académica prevalece un ejercicio académico "liberal" por el cual las y los profesores se contratan de manera temporaria por fuera de la planta regular de la institución (Pérez Centeno, 2015). De acuerdo con las entrevistas realizadas a docentes de posgrado de un programa no arancelado, la falta de remuneración específica y la intensificación de tareas que supone hace que el dictado de los cursos quede sujeto a las posibilidades y/o voluntades individuales. Sin embargo, la mayoría de quienes fueron entrevistados señaló que la decisión de trabajar ad honorem obedece a que la tarea docente en posgrado es una importante fuente de prestigio y se convierte en una posibilidad de vincular docencia e investigación.

Las políticas de evaluación constituyen uno de los principales dispositivos de la nueva gestión pública para la regulación del campo universitario. También desde la década de 1990, en Argentina, las políticas universitarias de evaluación se erigieron como: 1) instrumentos de redistribución presupuestaria, 2) mecanismos de división, clasificación y distinción de los actores en el campo universitario y 3 ) dispositivo de regulación de las prácticas de los actores. Además de las evaluaciones institucionales y la acreditación de carreras de grado y posgrado, las políticas de retribución económica según desempeño orientaron las pautas de evaluación del trabajo docente universitario. El Programa de Incentivo a los Docentes Investigadores, implementado desde el año 1993 en nuestro país, continúa vigente, aunque su mayor relevancia reside en la dimensión simbólica más que en la material. Actualmente, el complemento económico es irrisorio en términos monetarios, pero ocupar las más altas categorías del Programa ("ser" - más que "tener"- Categoría I o II) conlleva una importante carga de reconocimiento y prestigio en la comunidad académica. Los procesos de evaluación imperantes en el capitalismo académico se caracterizan por estar orientados por criterios productivistas y tecnocráticos, centrados en formatos electrónicos y 
parámetros cuantitativos que sumergen a los y las docentes en un "permanente completar casilleros", en un sentirse "evaluados hasta el extremo" y en un sentir que "hada alcanza, siempre te piden más", según relatan los entrevistados (Entrevistas semi-estructuradas, docentes CExN, 2013).

Hay docentes que sostienen que los procedimientos de evaluación del trabajo constituyen una oportunidad para desarrollar propuestas colectivas y consensuar criterios. Sin embargo, la mayoría señala que su carácter fragmentario y desarticulado exige un permanente "amoldarse" y "ajustarse" a cada requerimiento y, al mismo tiempo, produce un importante cúmulo de información que suele superponerse y no siempre es utilizada para la toma de decisiones a nivel institucional. Además, hay que reconocer que el discurso de la evaluación como garante de objetividad y justo reconocimiento de méritos se ha instalado en el mundo universitario y, más allá de las resistencias y cuestionamientos, constituye un principio orientador de las prácticas al definir lo que resulta redituable para mantener o mejorar la posición en el espacio universitario.

Finalmente, la expansión de la EaD, mediada por las tecnologías de la información y la comunicación (TIC) a la que se asiste en las últimas décadas a nivel mundial, responde a distintos factores entre los que se pueden mencionar la masividad, la necesidad de alcanzar a poblaciones más amplias que no pueden acceder a centros universitarios bajo la modalidad presencial, así como a las demandas del mercado.

En Argentina, desde fines de la década de 1990, las universidades comienzan a incorporar las TIC no solo para la gestión administrativa de sus actividades, sino también para brindar propuestas educativas virtuales (Guido y Versino, 2012). Un diagnóstico reciente sobre el sistema de educación superior argentino promovido por el Núcleo de Estudios e Investigaciones en Educación Superior del Mercosur (NEIES) señala que, desde el año 2000, la oferta de EaD ha experimentado un desarrollo exponencial que careció de políticas de planificación que permitan articular la oferta de las instituciones, lo que se deduce de la superposición de la oferta académica que se verifica en las diferentes regiones (Fernández Lamarra et al., 2018). En esa expansión, las carreras de grado crecieron un 64 \%: pasaron de 30 en 2000 a 192 en 2015; mientras que la oferta a distancia de posgrado se extendió un 95 \%: tenía seis carreras en 2002 y presenta 57 en 2015 . Al mismo tiempo, durante el período 2000-2015, se invierte la relación entre la cantidad de carreras a distancia que ofrece cada sector, ya que en el 2000 el sistema en su conjunto tenía 22 carreras a distancia de gestión pública y 14 de gestión privada, e inversamente, en 2015, hay 123 carreras a distancia en el subsistema estatal y 126 en el subsistema privado (Fernández Lamarra et al., 2018).

A la falta de políticas de planificación se le adiciona la falta de políticas de financiamiento específico que permita garantizar las condiciones para su adecuado desarrollo. Actualmente, la Resolución Ministerial 2641-E/2017, que regula la educación superior a distancia, establece la creación y evaluación en cada universidad de un Sistema Institucional de Educación a Distancia (SIED) que aglutine las ofertas académicas bajo la opción pedagógica y didáctica a distancia. Esta propuesta se instala en ámbitos institucionales que presentan particulares y desiguales niveles de desarrollo de la EaD en cuanto a personal técnico y especializado, recursos técnicos e infraestructura 
digital. En las universidades en que estas condiciones no están garantizadas, la implementación sin financiamiento de esta política de EaD hace que en las instituciones se apele a la voluntad y esfuerzo personal del plantel docente y técnico.

\section{Vivir on-line}

"Ya ni quiero abrir el mail. Un día que no lo abrís y tenés la casilla llena. Responder mail ya es todo un trabajo... Y satura" (Conversación informal con Profesor CSyH, 2019); "Ahora, estamos en período de residencias. Así que recibo mensajes todo el tiempo. Todo el tiempo, cualquier día y a cualquier hora tengo mensajes de WhatsApp de los estudiantes" (Conversación con Auxiliar de docencia, CSyH, 2019); "Zoom, Meet, correo electrónico, WhatsApp... Este año quedé pasada de pantalla" (Conversación informal, Profesora CSyH, 2020).

Los enunciados anteriores nos sumergen de lleno en una de las tendencias que desde hace décadas viene ganando protagonismo en las universidades y que la situación de pandemia por COVID-19 durante el año 2020 aceleró abruptamente: la virtualización de las actividades académicas. Hay que reconocer que el uso de tecnologías no es un fenómeno nuevo en las universidades y que los y las académicos/as siempre han empleado algún tipo de tecnología como medio de trabajo. Lo novedoso en estos tiempos es la centralidad de las tecnologías digitales y la virtualización de las actividades en las universidades que han provocado cambios cualitativos en los procesos y condiciones de trabajo y en los modos de comunicación entre docentes (Allmer, 2018).

Al respecto, puede señalarse que la dinámica de cambios que impulsan las TIC se hace presente a diferentes niveles del campo de la educación superior. Por un lado, a nivel de los sistemas, conformando un subsector cada vez mayor a partir de la irrupción de nuevas ofertas institucionales de EaD y de la virtualización de distintos aspectos de las propuestas presenciales. Como se dijo en el apartado anterior, el acelerado desarrollo de estas modalidades puede entenderse como fuente de democratización de conocimientos al ampliar el alcance de poblaciones excluidas de la educación superior, pero también es necesario reconocer su vinculación con la globalización mercantil de la universidad (Sousa Santos, 2005) y las condiciones de desigualdad -entre sistemas nacionales, universidades y sujetos- en que se las incorpora. Por otro lado, a nivel institucional, la creciente virtualización está promoviendo modificaciones en las formas de gestión de las universidades y sus procedimientos administrativos que conllevan también cambios en los perfiles institucionales y las ofertas académicas. Finalmente, a nivel de los sujetos, las tecnologías digitales cobran protagonismo en las ofertas académicas de EaD, pero también en las propuestas académicas presenciales a través de la introducción de recursos y plataformas digitales en las prácticas de enseñanza, en las formas de trabajo académico colaborativo, en la comunicación cotidiana con estudiantes y colegas, etc.

La virtualización del trabajo docente en la universidad experimentó una insospechada aceleración a partir de los procesos de enseñanza remota de emergencia llevados adelante durante 
la pandemia por COVID-19. Pero, como se advierte en las investigaciones que sirven de sustento a esta comunicación, la virtualización del trabajo académico constituye una tendencia creciente en los últimos años a nivel mundial. El análisis de entrevistas realizadas a docentes de carreras de grado y posgrado presenciales de universidades argentinas en el período previo a la pandemia permite reconocer la centralidad de las actividades virtuales en su trabajo docente cotidiano y que contribuyen a desdibujar las fronteras entre el espacio laboral y el privado o familiar, descentrando no solo el espacio de enseñanza y aprendizaje de los confines del aula, sino también los tiempos que exceden los límites de la jornada laboral prescripta. Si bien hay quienes dicen valorar la autonomía que brinda el workhome, la mayoría experimenta el teletrabajo como una forma de intensificación del trabajo docente, un trabajo a demanda que requiere estar constantemente conectado.

Como sostiene Sennett (2006), el imperativo de la conectividad coloca ante la exigencia de atención, vigilancia y disponibilidad permanente. En su análisis de la universidad británica, Gill (2015) hace referencia a una "academia sin paredes" que exige estar a/ways on. Así, si bien la literatura sobre el tema ya lo venía señalando, la virtualización de la universidad en tiempos de pandemia invita a profundizar el estudio de las relaciones entre vida y trabajo, tiempo laboral y tiempo de ocio, espacio privado y espacio público, workhome y trabajos de cuidado, así como las consecuencias subjetivas, vinculares y en la salud del teletrabajo atendiendo a la forma diferencial que afecta a varones y mujeres.

\section{El tiempo, divino tesoro}

"El tiempo que uno pierde completando casilleros... y cada proceso de evaluación tiene un formato distinto" (Entrevista a profesora, CSyH, 2013); "Pareciera que siempre falta más, siempre 'te corren la raya"' (Entrevista a profesora, CExN, 2013); "Vivo ajustada en los tiempos" (Entrevista a profesora, CExN, 2013); "Nunca hay tiempo" (Entrevista a profesora, CExN, 2013); "Yo quisiera dedicar más tiempo a la investigación, pero a veces es imposible" (Entrevista a profesora, CExN, 2013).

Gill (2018) sostiene que el tiempo es quizás la mayor fuente de disputas, ansiedad y estrés en la academia debido a la exigencia creciente de "hacer más con menos" en una cultura que pide "estar siempre activo" y que se encuentra marcada por un constante estado de "emergencia como regla". Recuperando la literatura especializada a nivel internacional y a partir de sus estudios sobre la universidad británica, la autora señala que las y los académicas/os anhelan más tiempo para investigar, no tienen suficiente tiempo para leer, dedican demasiado tiempo al trabajo, no logran adaptar su trabajo al tiempo de la jornada laboral, no tienen tiempo para cualquier cosa fuera del trabajo y luego están sujetos al mito de que son ricos en tiempo y ociosos. Una permanente sensación de "no disponer de tiempo", de "necesitar más tiempo", de "vivir ajustada en los tiempos", como manifestó la mayoría de quienes fueron entrevistados. Como sintetiza Pérez Bustos (2019) en su análisis de las temporalidades de la ciencia y los actuales estándares de medición que regulan la 
producción de conocimiento científico y sus consecuencias en los sujetos, en la academia, impera un permanente sentir que "mi tiempo ya no es mío".

Lo anterior nos lleva a formular una breve disquisición sobre el concepto de tiempo en la medida en que constituye un importante regulador de la vida social. Desde una perspectiva sociológica, Norbert Elias (1989) concibe el tiempo como una construcción sociohistórica, un "dato social" que sirve de orientación para las sociedades y que ha experimentado, a lo largo del proceso civilizatorio, una evolución hacia lo que denomina la "autodisciplina del tiempo". Elias define al tiempo como un aspecto del proceso de la civilización y por tanto del proceso de desarrollo de las estructuras social e individual que cumple las funciones de orientación social y regulación de las conductas y las sensibilidades humanas. Sintéticamente, el autor señala tres niveles de integración en la evolución de la determinación del tiempo: a) el nivel natural, el de la Naturaleza, donde la organización humana está regida por los fenómenos naturales recurrentes e inhumanos (por ejemplo, los movimientos de los astros) y los grupos vivencian una determinación del tiempo intermitente, puntual, pasiva y relajada; b) el nivel social, integrado en el nivel anterior, caracterizado por la construcción de instrumentos humanos para determinar y regular el tiempo (por ejemplo, relojes, calendarios); c) el nivel individual, en el que la determinación del tiempo se presenta como autodisciplina socialmente aprendida e introyectada en la estructura de personalidad. Este último nivel es el que impera, según el autor, en las sociedades urbanizadas e industrializadas más complejas y diferenciales como las actuales, en las que la determinación del tiempo es exhaustiva y activa, con sofisticados instrumentos de medición y prevalencia de un sentimiento del tiempo como inexorable. Así, el tiempo aparece como una unidad de medida y de sentido de todos los acontecimientos junto a la interiorización de una severa autodisciplina del tiempo. Elias (1989) aclara que las diferencias entre las etapas no estriban en que las autocoacciones estén presentes en un caso y ausentes en el otro. Lo que cambia es la relación entre coacciones externas e internas y, especialmente, el modelo de autodisciplina y el tipo de su implantación.

Este proceso de determinación del tiempo, autodisciplina socialmente aprendida, resulta fundamental para comprender las relaciones entre tiempo, trabajo y subjetividad en las actuales formas de organización del trabajo docente en la universidad. Un dato significativo que surge de las entrevistas es que, a pesar de sentir que el tiempo nunca alcanza ante la intensificación de las tareas, la mayoría de los y las docentes se involucran con ellas y asumen cada vez más responsabilidades. Y esto por distintas razones: "hay que ocupar esos espacios", porque "es lo que me gusta", porque "es necesario". Antunes (2001), en su análisis sobre las mutaciones actuales en el mundo del trabajo, alude al "involucramiento cooptado", que le posibilita al capital apropiarse del saber y del hacer del trabajo. Zangaro (2010) emplea los conceptos de "subjetividad implicada" y "alienación voluntaria" para referirse a este fenómeno. Tales categorías expresan un proceso de subjetivación en el que ya no resultan imprescindibles las obligaciones hetero-impuestas a las y los trabajadoras/es, en este caso, docentes universitarios, quienes se las autoimponen. Para lograr trabajadores autoexigidos, para configurar estas "subjetividades implicadas", se torna necesaria la puesta en marcha de un aparato justificativo cuyas razones, ancladas en la realidad cotidiana de los 
sujetos, permita movilizarlos hacia lo que se espera de ellos (Boltanski y Chiapello, 2002). El discurso de la autonomía y la flexibilidad como sinónimos de libertad puede considerarse otra de esas razones. Como se advierte en el siguiente apartado, las TIC han contribuido con ese discurso al presentar "zonas de autonomía" o el disfrute de cierta libertad de elección -de espacios y tiempos-, aunque exponiendo más directamente a las y los docentes a la presión de la demanda y a la presión de los plazos.

\section{Vida laboral, vida personal}

"Disculpas la tardanza en responder. Han sido y están siendo unas semanas intensas y cargadas de trabajo. Estoy saturado de cosas" (Correo electrónico de un profesor, CSyH, 2019);

"Entre la vida familiar, que nos vamos poniendo viejas las integrantes y se complica mucho la salud, y la vida institucional, en la que se dio una situación muy decepcionante, que fue un shock de estrés... del cual aún me recupero" (Correo electrónico de una profesora, CSyH, 2018); "Tengo una semi-dedicación porque quiero tener una semi-vida" (Entrevista a Auxiliar de docencia, CEyA, 2012).

Los fragmentos anteriores convocan a pensar la universidad como espacio laboral atravesado por la precariedad que actualmente atraviesa a todo el cuerpo social presentándose diferencialmente según sectores y colectivos (Castillo y Moré, 2018). Como sostiene Gill (2015), la precariedad es una de las experiencias definitorias de la vida académica contemporánea, particularmente (aunque no de forma exclusiva) entre quienes son más jóvenes y/o se encuentran en los inicios de su carrera académica. Un componente importante de las situaciones de precariedad es el incremento de formas de contratación laboral a tiempo parcial y de forma temporaria al que asisten los sistemas universitarios en distintas latitudes en consonancia con los cambios acontecidos en el mundo del trabajo en general (Castel, 2015; Sennett, 2006). También el trabajo no remunerado. El trabajo ad honorem en la universidad incluye el trabajo no remunerado en el desempeño de cargos docentes, pero también la creciente multiplicidad de tareas necesarias para el funcionamiento de la actividad académica (conformación de comisiones curriculares, consejos asesores, coordinación de áreas, departamentos y carreras, jurados de tesis y concursos docentes, etc.) que se realizan en el marco de las mismas dedicaciones horarias.

El despliegue de carreras nómades, intermitentes e inciertas hacen que las trayectorias graduales en una misma institución resulten inasibles para quienes recién se inician como docentes en la universidad contemporánea. En la actualidad, las trayectorias laborales de docentes noveles se van construyendo en el marco de los horizontes acotados de los períodos de becas y la renovación de contratos. Esto hace que surjan como alternativas los empleos fuera de la universidad, el abandono temporario de la docencia universitaria, los trabajos por cuenta propia, etc. (Walker, 2017). La cuestión del placer, del "me gusta", "es lo que me gusta", "es lo que deseo", ofrece una convincente justificación del propio estatus, además de un mecanismo disciplinario para 
tolerar no solo la incertidumbre y la autoexplotación, sino también para mantenerse en una situación precaria y aun así no abandonarla. Todo esto acontece en un contexto en el que estamos profunda y apasionadamente apegados al trabajo no siendo capaces, muchas veces, de trazar una separación entre nuestro trabajo y nosotros y nosotras mismas. De ahí la necesidad de pensar cómo algunos placeres del trabajo académico nos atan cada vez más a un régimen neoliberal con costos cada vez mayores (Gill, 2015).

En las entrevistas realizadas, la necesidad de "salir de la universidad" fue recurrente entre docentes que ocupan posiciones con estabilidad laboral (cargos ordinarios) y amplia dedicación en la institución (exclusiva) o en aquellos que tienen su actividad principal fuera de la universidad (profesiones liberales). Una docente JTP, con dedicación exclusiva del área de CSyH, afirma: "Necesito salir para chocarme con la realidad" (Entrevista con JTP, CSyH, 2019). Por su parte, un profesor de la misma área ya retirado relata:

Yo me jubilé el año pasado. Sigo vinculado a la universidad, a través de la investigación y dando clases de posgrado de vez en cuando (...). Tengo amigos que se jubilaron y cortaron absolutamente, estaban esperando jubilarse para irse. No volvieron más. Pero esos casos que conozco son de personas que tuvieron dedicación exclusiva en la universidad. Yo siempre tuve otras actividades, otros trabajos... creo que por eso no me harté. La universidad no era mi centro. (Conversación con profesor jubilado, CEyA, 2018)

Esta última cuestión referida a la universidad como "centro" de la vida alude a las relaciones entre vida y trabajo, las cuales ocupan un lugar destacado como objeto de estudio de la sociología del trabajo en los últimos años (Castillo, 2015). Y es que, en la actualidad, el trabajo ha invadido todas las esferas de la vida con los riesgos que ello conlleva. Cuando el trabajo ocupa un porcentaje muy importante del tiempo para asegurar el consumo de la familia y los seres humanos devienen animales laborans, su participación en la vida política y en las actividades públicas se ve reducida (Neffa, 2003).

El ejercicio del trabajo docente durante la situación de emergencia sanitaria por la pandemia profundizó la fusión entre vida personal y trabajo, amalgamando tiempos laborales con tareas de cuidado y reduciendo significativamente los tiempos de ocio y descanso. Esta aceleración abrupta de una tendencia que ya venía ganando terreno hace tiempo, en el campo universitario y otras esferas del mundo del trabajo, merece ser estudiada con especial atención en las consecuencias subjetivas, vinculares y en la salud psicofísica. La pandemia hizo más evidente aquello que ya se venía señalando en el ámbito universitario: que "tener una vida" parece cada vez más un logro (Gill, 2018).

\section{Reflexiones finales}

La universidad se presenta como un espacio heterogéneo no solo por el mapa multicolor que trazan las distintas matrices disciplinares (Becher, 2001) y la coexistencia de disímiles tradiciones teóricas, epistemológicas y metodológicas que anidan en ellas, sino también por las particulares culturas organizacionales del conglomerado de unidades académicas que la componen, 
las formas de organización del trabajo que se tornan imperantes en cada momento histórico y su relación con los cambios operados en el mundo del trabajo en general y su vinculación con el Estado y la sociedad. Esta heterogeneidad da lugar a un entramado de distintas dimensiones que atraviesan el espacio universitario y configuran distintas experiencias de trabajo.

En el contexto del capitalismo académico, pensar la universidad contemporánea como espacio laboral supone comprender sus lógicas y dinámicas en estrecha relación con los cambios operados en el mundo del trabajo en general: formas de contratación laboral caracterizados por el tiempo parcial y contratos temporarios, el desarrollo de carreras nómades, la virtualización de las actividades, el control de los resultados, etc. La racionalidad empresarial se erige como el modelo de organización universitaria que busca invisibilizar a la universidad como espacio público, como lugar de encuentro y controversia entre muchas y potenciales identidades. La autonomía y flexibilidad, la transparencia de los procesos y la objetividad de los resultados y la productividad como medida del trabajo se conjugan en un entramado discursivo que pretende trabajadores automotivados y emprendedores, gestores de sus propias trayectorias y carreras, competentes, productivos.

Una de las constataciones que permitió "girar las lentes" para comprender el trabajo docente universitario es la de reconocer la desigualdad que atraviesa las experiencias de trabajo en la universidad. Más allá de la homogeneidad operada por las nominaciones oficiales y las regulaciones, incluso al interior de una misma categoría, en el sistema oficial de jerarquías, existe una diversidad de situaciones, condiciones y demandas no siempre explícitas, reconocidas o formalizadas.

Los dispositivos de regulación de la nueva gestión pública configuran distintas experiencias dando lugar a prácticas estratégicas, adaptativas o de resistencia en el marco de las desiguales formas de ejercer el trabajo que se expresan en múltiples formas de habitar la universidad contemporánea y que requieren ser indagadas en profundidad. En este escenario, se modifican no solo las condiciones y formas de organización del trabajo, sino su propia naturaleza y los sentidos que los sujetos le atribuyen. Sentidos construidos en las experiencias cotidianas del trabajo que operan como fuente de satisfacción o de malestar.

En el espacio laboral que es la universidad, se suele escuchar en las conversaciones entre colegas: "Pero bueno... somos unos privilegiados", en un intento de balancear con esta expresión de cierre todo aquello que la experiencia de trabajo nos provoca. La apelación a las posibilidades de autonomía, creatividad, la flexibilidad de los horarios y el home office pareciera contrarrestar -como si no tuviesen algún tipo de conexión- la intensificación de tareas, la presión por los plazos y tiempos, el hartazgo y los sinsentidos experimentados en lo cotidiano por el creciente control por resultados. ¿Qué es lo que se siente como un privilegio? ¿Qué se entiende por privilegio? Desarmar nuestras representaciones, sentires y decires sobre nuestro trabajo docente en la universidad parece ser un buen comienzo para visibilizar los sentidos que hemos ido construyendo. En principio, concebir a la universidad como espacio laboral -atravesado por lógicas y dinámicas presentes en otros campos- y reconocernos como trabajadores y trabajadoras - un grupo social que 
históricamente se ha resistido a hacerlo- puede ser un importante inicio. Y, a partir de allí, girar las lentes, agudizar la mirada en aquello que acontece en lo cotidiano y buscar los modos de visibilizar y problematizar las actuales formas de ejercicio del trabajo docente en la universidad contemporánea. Y es que la narración de la experiencia, más que una mera expresión o enunciación a posteriori de ella, se constituye en un espacio privilegiado para su configuración misma como experiencia colectiva.

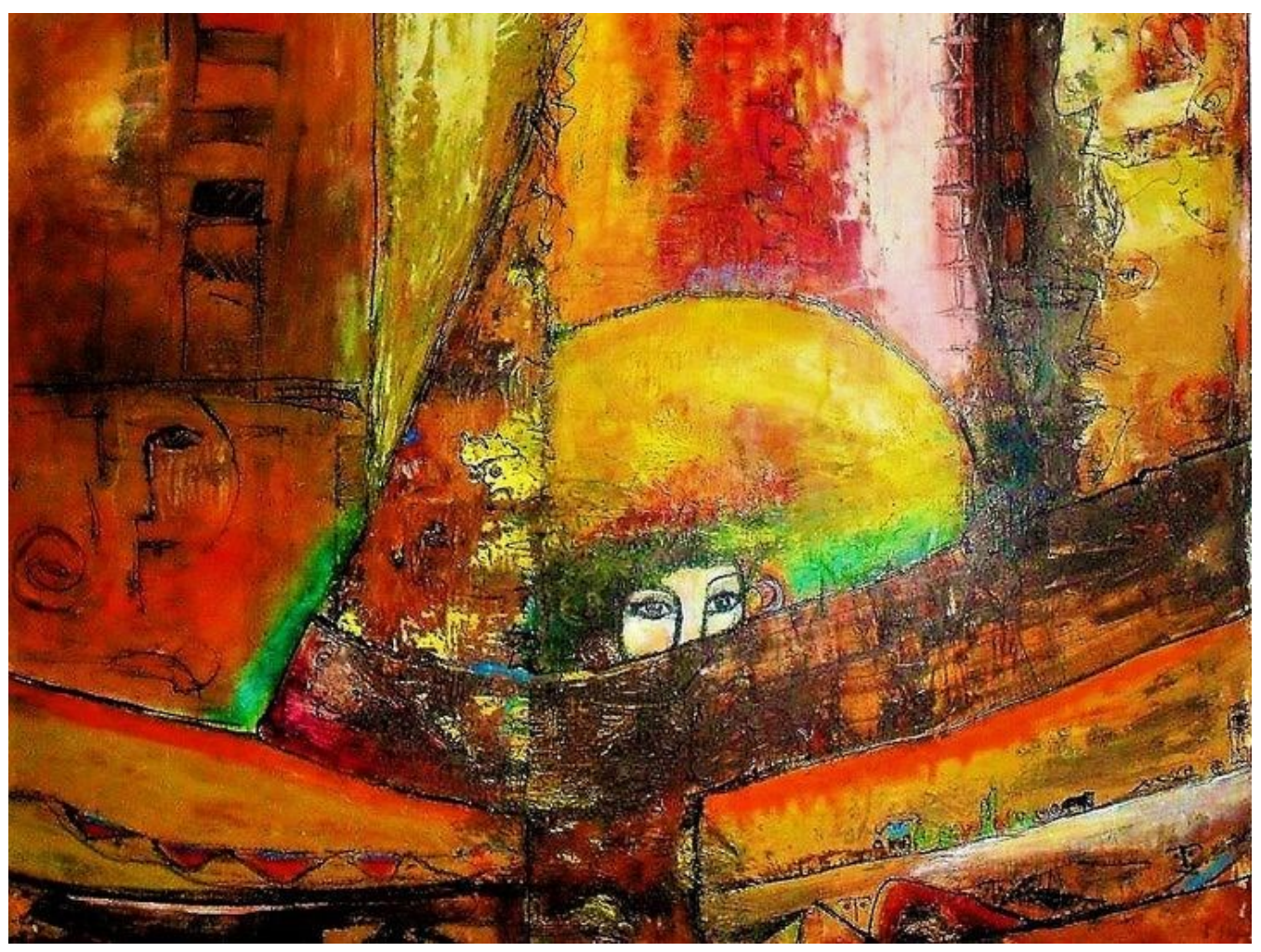

Fiesta de la Pachamama, técnica mixta. Adriana Chavarri

\footnotetext{
' Investigación doctoral titulada "Evaluación y trabajo docente en la universidad: políticas, prácticas y actores. Estudio de casos en Argentina y España" (2011-2013). Red de Investigación "Dilemas de nuevas culturas de producción de conocimiento. Los postgrados en Argentina, Brasil y Paraguay en el contexto de la evaluación de la calidad de la Educación Superior". Núcleo de Estudios e Investigaciones en Educación Superior del Sector Educativo del MERCOSUR. M.E-SPU (Primera etapa: 2013-2015. Segunda etapa: 2015-2017). Proyecto Grupal de Investigación (PGI) "Universidad y formación de profesionales y trabajadores de la educación: funciones, sujetos y prácticas", SCCYT-Universidad Nacional del Sur (2016-2019).

ii Cada extracto está acompañado por una referencia que indica el tipo de material empírico, categoría docente, área disciplinar y año. Las áreas disciplinares son: Ciencias Sociales y Humanidades (CSyH), Ciencias Exactas y Naturales (CExN) y Ciencias Económicas y de la Administración (CEyA).

iii Expresión coloquial: "no hay dinero".
} 


\section{Bibliografía}

Antunes, R. (2001). ¿Adiós al trabajo? Ensayo sobre la metamorfosis y la centralidad del mundo del trabajo. Cortez Editora.

Allmer, T. (2018). Theorising and Analysing Academic Labour. TripleC: Communication, Capitalism \& Critique, 16(1), 49-77.

Araujo, S. (2003). Universidad, investigación e incentivos. La cara oscura. Ediciones Al Margen.

Barnett, R. (2002). Claves para entender la universidad. En una era de supercomplejidad. Pomares.

Becher, T. (2001). Tribus y territorios académicos. La indagación intelectual y las culturas de las disciplinas. Gedisa.

Beltrán Villalba, M. (2013). La hermenéutica del sentido de las "cosas sociales". ARBOR Ciencia, Pensamiento y Cultura, 189(761), 1-8.

Boltanski, L. y Chiapello, È. (2002). El nuevo espíritu del capitalismo. Ediciones Akal.

Bourdieu, P. (2008). Homo Academicus. Siglo XXI Editores.

Boyer, E., Altbach, P. y Whitelaw, M. J. (1994) The Academic Profession. An International Perspective. A Special report. The Carnegie Foundation for the Advancement of Teaching. NJ.

Brunner, J. (1985). Universidad y sociedad en América Latina: un esquema de interpretación. UNESCO-CRESALC. Brunner, J. J. y Flisfisch, A. (1983). Los intelectuales y las instituciones de la cultura. FLACSO.

Carli, S. (2016). Deconstruir la profesión académica: tendencias globales y figuras históricas. Una exploración de las biografías académicas de profesoras universitarias. Revista Propuesta Educativa, (45), 81-90.

Castel, R. (2015). Las trampas de la exclusión. Trabajo y utilidad social. Editorial Topia.

Castillo, J. (2015). La invasión del trabajo en la vida. Del "trabajador ideal" a la vida real. Catarata.

Castillo, J. y Moré, P. (2018). Universidad precaria, universidad sin futuro. Fuhem.

Chiroleu, A. (2002). La profesión académica en Argentina. Boletín PROEALC, Síntesis Especial América Latina.

Clark, B. (1983). El sistema de educación superior: Una visión comparativa de la organización académica. Nueva Imagen.

Elias, N. (1989). Sobre el tiempo. Fondo de cultura económica.

Enders, J. y Kaulisch, M. (2006). The Binding and Unbinding of Academic Careers. En U. Teichler (Ed.), The Formative Years of Scholars (pp. 85-96). Portland Press.

Fernández Lamarra, N., Pérez Centeno, C., Marquina, M. y Aiello, M. (2018). La educación superior universitaria argentina: situación actual en el contexto regional. Editorial EDUNTREF.

García de Fanelli, A. M. (2008). Profesión académica en la Argentina: Carrera e incentivos a los docentes en las Universidades Nacionales. CEDES.

Gil Antón, M. (2013). La monetarización de la profesión académica en México: Un cuarto de siglo de transferencias monetarias condicionadas. Espacios en Blanco. Revista de Educación, (23), 157-186.

Gill, R. (2015). Rompiendo el silencio. Las heridas ocultas de la universidad neoliberal. Arxius de Ciencias Sociales, (32), 45-58.

Gill, R. (2018). Beyond Individualism: The Psychosocial Life of the Neoliberal University. En: M. Spooner y J. McNinch (Eds.), Dissident knowledge in higher education (pp. 193-216). University of Regina Press.

Guber, R. (2004). El salvaje metropolitano. Reconstrucción del conocimiento social en el trabajo de campo. Editorial Paidós.

Guido, L. y Versino, M. (2012). La Educación Virtual en las Universidades Argentinas. IEC-CONADU.

Heller, A. (1987). Sociología de la vida cotidiana. Ediciones Península. 
Ibarra Colado, E. (2003). Capitalismo académico y globalización: La universidad reinventada. Revista Educação \& Sociedade, 24(84), 1059-1069.

Krotsch, P. (2001). Educación superior y reformas comparadas. Universidad Nacional de Quilmes.

Llomovatte, S. y Wischnevsky, J. (1998). Condiciones laborales de los docentes universitarios en los 90. Algunos resultados de investigación. Revista Praxis Educativa, 3(1), 15-26.

Marquina, M. (2013). ¿Hay una profesión académica argentina? Avances y reflexiones sobre un objeto en construcción. Revista Pensamiento Universitario, 15(15), 35-58.

Martínez, D. (2013). El trabajo en la universidad. Pequeña introducción a un texto para dar batalla. Revista Espacios en Blanco, (23), 45-72.

Musselin, C. (2007). The transformation of academic work: fact and analysis. Research \& Occasional Paper Series.

Nairdof, J. (2009). Los cambios en la cultura académica en las universidades públicas. EUdeBA.

Neffa, J. (2003). El trabajo humano. Asociación Trabajo y Sociedad.

Pérez Centeno, C. (2015). Cuatro modos del ejercicio académico en las universidades públicas argentinas. En N. Fernández Lamarra y C. Mundt, Sociedad, Procesos Educativos, Instituciones y Actores. Estudios de Política y Administración de la Educación II. EDUNTREF.

Perkin, H. (1984). The academic profession in the UK. En B. Clark (Ed.), The Academic Profession: National, Disciplinary and institutional Settings. University of California Press.

Robertson, S. (2013). Teachers' Work, Denationalisation, and Transformations in the Field of Symbolic Control: A Comparative Account. En J. Levin, T. Seddon y J. Ozga (Eds.), World Year Book of Education 2013. Routledge.

Rockwell, E. (2018). Temporalidad y cotidianeidad en las culturas escolares. Cuadernos de Antropología Social, (47), 21-32.

Saforcada, F. y Rodríguez Golisano, A. (2019). Privatización y mercantilización de la universidad en América Latina: sentidos y disputas. En F. Saforcada (Dir.), Tendencias de privatización y mercantilización de la universidad en América Latina. Los casos de Argentina, Chile, Perú y República Dominicana. IEC-CONAFU.

Sautu, R. (2005). Todo es teoría: objetivos y métodos de investigación. Lumiere.

Sennett, R. (2006). La cultura del nuevo capitalismo. Editorial Anagrama.

Silva Júnior, J. y Sguissardi, V. (2013). Universidade Pública Brasileira no Século XXI. Educación superior orientada para o mercado e intensificação do trabalho docente. Espacios en Blanco. Revista de educación, (23), 119-156.

Sousa Santos, B. (2005). La universidad en el siglo XXI. Para una reforma democrática y emancipadora de la universidad. Miño y Dávila.

Tello, A. M. (2008). Nuevas regulaciones en el trabajo docente. Reflexiones del Congreso Nacional por una Educación Superior Democrática, Popular, Emancipadora y Latinoamericana. Hacia la construcción de un nuevo movimiento nacional de trabajadores docentes universitarios. Edición ADIUC-AGD-COAD.

Verger, A. y Normand, R. (2015). Nueva gestión pública y educación: Elementos teóricos y conceptuales para el estudio de un modelo de reforma educativa global. Educação \& Sociedade, 36(132), 599-622.

Wright Mills, C. (1994). La imaginación sociológica. Fondo de Cultura Económica.

Zangaro, M. (2010). Subjetividad y trabajo: el management como dispositivo de gobierno. Revista Trabajo y Sociedad, XV (16), 163-177. 